\title{
Review
}

\section{Response of tumour cells to hypoxia: role of p53 and NFkB}

\author{
J A Royds, S K Dower, E E Qwarnstrom, C E Lewis
}

\begin{abstract}
Hypoxia is present in several areas of malignant tumours and is thought to result from an inadequate rate of tumour angiogenesis, vascular collapse, or both. The presence and extent of these hypoxic tumour microenvironments have recently been shown to influence tumour progression by regulating both tumour cell survival and the expression of key angiogenic molecules. Recent studies have suggested that mutations in the tumour suppressor gene, p53, may play an important role in regulating the adaptive response of tumour cells to hypoxia by enhancing their survival and release of proangiogenic factors such as vascular endothelial growth factor. It has even been suggested that hypoxia may select for the survival of the more malignant clones harbouring such genetic defects as mutations in p53. Recently, the transcription factor, NFkB, has also been implicated as a novel mediator of the effects of hypoxia and reoxygenation in tumour cells. This article reviews some of the molecular mechanisms subserving the responses of tumour cells to hypoxic stress, particularly the role and relation of $\mathrm{NFkB}$ and p53 in regulating this phenomenon.

(F Clin Pathol: Mol Pathol 1998;51:55-61)
\end{abstract}

Institute of Cancer Studies, University of Sheffield Medical

School, Sheffield S10

2RX, UK

J A Royds

Department of

Medicine \&

Pharmacology,

University of Sheffield

Medical School

$S \mathrm{~K}$ Dower

E E Qwarnstrom

Department of Pathology, University of Sheffield Medical

School

C E Lewis

Correspondence to: Dr Royds.

Accepted for publication 21 November 1997 partially collapses. The ability of tumo to survive at low oxygen tension is clinice relevant, as hypoxic tumour cells are resistant to radiotherapy and chemotherapy than their well oxygenated counterparts, and are capable of stimulating angiogenesis by the release of several growth factors for endothelial cells. This may explain, in part, why patients with extensively hypoxic tumours have a worse prognosis than those with well oxygenated tumours. ${ }^{1}$

Angiogenesis is thought to be driven largely by hypoxia in tumours and is particularly relevant to metastatic spread, as primary tumour cells enter the circulation via these new blood vessels and micrometastases must attract new capillaries if they are to grow beyond 2-3 $\mathrm{mm}^{3}{ }^{2}$ The rate of angiogenesis at different sites within solid tumours is controlled by the local balance of stimulatory and inhibitory factors, and involves activation of endothelial cell proliferation, migration, and differentiation to form new capillary-like structures. Inhibitory influences predominate in most normal adult healthy tissues, but tumour cells are usually proangiogenic as they need to attract a new vasculature to provide oxygen and growth factors to sustain their high proliferative activity. ${ }^{3}$

Many of the molecular mechanisms regulating angiogenesis have now been outlined, ${ }^{4}$ and recent advances have revealed the central role of the proangiogenic cytokine, vascular endothelial growth factor (VEGF; also known as vascular permeability factor (VPF)). Increased expression of VEGF is found in hypoxic areas of many solid tumours. ${ }^{5}$ This pivotal mediator of tumour angiogenesis is produced by malignant cells and macrophages, and binds mainly to its receptors fit- 1 or flk-1 on endothelial cells in tumours to promote their proliferation and migration. VEGF expression by breast carcinoma cells confers a growth advantage on tumour cells in vivo but not in vitro, inferring that this effect may be mediated by VEGF induced tumour vascularisation. ${ }^{6}$ Moreover, inhibition of VEGF suppresses metastasis by a mechanism distinct from its effects on primary tumour growth. ${ }^{7}$

VEGF is encoded by a single gene that generates isoforms of the protein by differential splicing giving at least two secreted $\left(\mathrm{VEGF}_{121}\right.$ and $\left.\mathrm{VEGF}_{165}\right)$ and two bound $\left(\mathrm{VEGF}_{189}\right.$ and $\mathrm{VEGF}_{206}$ ) forms. Transgenic mice lacking a single VEGF allele die in utero from impaired blood vessel formation, indicating a dose dependent importance for VEGF in angiogenesis. Furthermore, VEGF null embryonic stem 


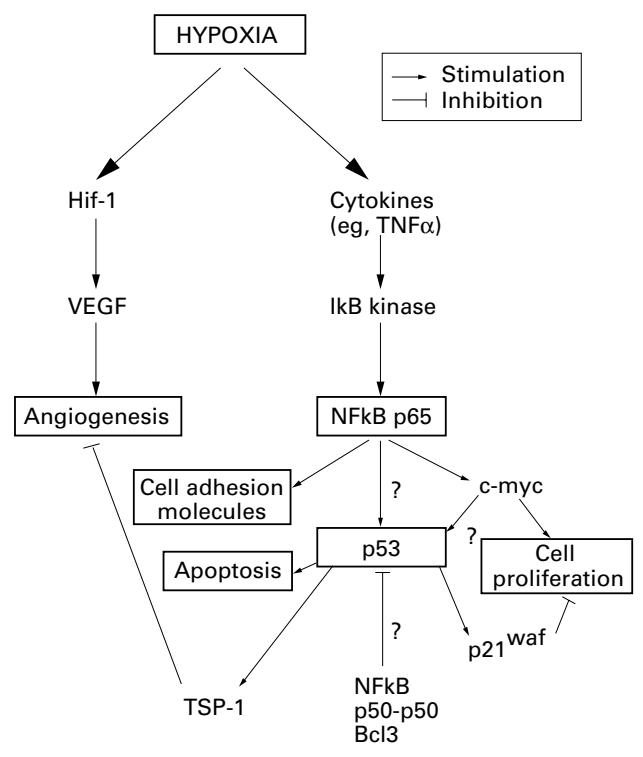

Figure 1 Potential model of the molecular mechanisms subserving response of tumour cells to hypoxia. Hypoxia activates expression of the VEGF gene via the hif-1 response element in its promoter. This proangiogenic activity is then counterbalanced by the production of TSP-1, the downstream effector of p53. p53 itself is induced in response to hypoxia via the NFkB pathway. Cellular proliferation is inhibited by the action of CDKIs (such as p2 $1^{\text {waf }}$ ) and apoptosis is increased; both of which are also induced by p53.

cells have a significantly reduced ability to produce tumours in nude mice compared to wildtype cells. ${ }^{8}$

The VEGF family is composed of three members (VEGF, VEGFB, and VEGFC) plus the related placental growth factor, PIGF, all of which are encoded on different human chromosomes. ${ }^{9}$ The expression of these peptides is differentially regulated, with each performing distinct but related functions.

\section{Cellular responses to hypoxia}

There are three main types of cellular response to hypoxia in normal tissues: inhibition of cellular proliferation; induction of apoptosis; and the release of proangiogenic cytokines. The molecular mechanisms of cell death from hypoxia are poorly understood but are thought to involve both apoptosis and necrosis in several cell types (fig 1). ${ }^{10}$ Tumour hypoxia is characteristically transient in nature and the mode of cell death that follows reperfusion after ischaemia is thought to be primarily apoptosis. $\mathrm{Bcl}-2$ and $\mathrm{Bclx}_{\mathrm{L}}$ inhibit hypoxia induced cell death in a dose dependent manner, again suggesting the apoptotic route. Prolonged hypoxia, on the other hand, causes both necrosis and apoptosis. ${ }^{11}$

Human cells respond to fluctuations in environmental oxygen by the activation of specific mediators. One of these has attracted considerable attention to date - the transcription factor, hypoxia inducible factor 1 (Hif- 1 ) ${ }^{12} \mathrm{Hif}-1$ was first described as a DNA binding factor essential for functional hypoxia induced transcription of the erythropoietin gene. ${ }^{13}$ This DNA binding factor is rapidly induced by hypoxia and binds to the hypoxia responsive element (hre) of the gene promoters for many oxygen sensitive genes, such as VEGF, thereby activating gene transcription. Recent evidence has shown that another basic helix loop helix protein with a PAS domain (bHLH-PAS) with sequence homology to $\mathrm{Hif}-1$ also regulates VEGF expression, but with a tissue specific potency. ${ }^{14}$ Moreover, another hypoxia activated PAS domain transcription factor, endothelial PAS-1 (EPAS1), has also been characterised in endothelial cells. This mediates the stimulatory effect of hypoxia on the expression of the endothelial cell genes, particularly the tyrosine kinase, Tie- $2 .{ }^{15}$

\section{Response of tumour cells to hypoxia: role of p53}

Mutations in the tumour suppressor gene, p53, are the most common genetic defects in solid tumours. Most studies of the role of p53 protein in cancer have focused on its function in cell cycle control, drug resistance, and apoptosis. However, there is now increasing evidence that mutations in p53 play an important role in regulating the survival and angiogenic responses of tumour cells to hypoxia. Wildtype p53 acts as a transcriptional regulator of genes involved in cell cycle control such as the cyclin dependent kinase inhibitor $\mathrm{p} 21^{\text {waf }}$, and in apoptosis, bringing about homeostasis in conditions of cellular stress.

However, mutant p53 has recently been shown to confer a growth advantage on cells under hypoxic conditions and to accumulate in the nuclear compartment of cells under low oxygen conditions. ${ }^{16}$ Furthermore, in cervical cancer, hypoxia provides a selection pressure for tumour cells with reduced apoptotic potential (such as those bearing mutant p53). ${ }^{17}$ Our own studies using three dimensional cultures of human tumour cells (multicell spheroids, which have a profoundly lower concentration of oxygen in the central than the outer tumour cell layers) have shown that tumour cells bearing mutant p53 are able to sustain a longer period of cellular proliferation in hypoxic conditions than those with the wild-type gene (Royds JA, Lewis CE, unpublished data, 1997). Hypoxia gives a non-genotoxic stress that induces p53 activity, but there is evidence to suggest it is via a different pathway from the DNA damaging agents. ${ }^{18}$ Unlike most other tumour suppressor genes, p53 mutations are missense rather than loss of function mutations (such as truncations or deletions), and these usually persist, often becoming the dominant clone in metastases (Alcock A, Royds JA, unpublished observations, 1997). The fact that p53 mutations, once selected for by tumour signals such as hypoxia, are maintained both in primary and secondary tumours also suggests a positive phenotype for mutant $\mathrm{p} 53$. Evidence is now emerging to support this hypothesis. ${ }^{19}$

Long term survival in vivo of transformed cells with a defective apoptotic potential requires the activation of neoangiogenesis to augment an insufficient oxygen supply. p53 gene status has also been implicated in the regulation of tumour angiogenesis. It is well known that hypoxia induces the angiogenic potential of tumours by stimulating the 
A

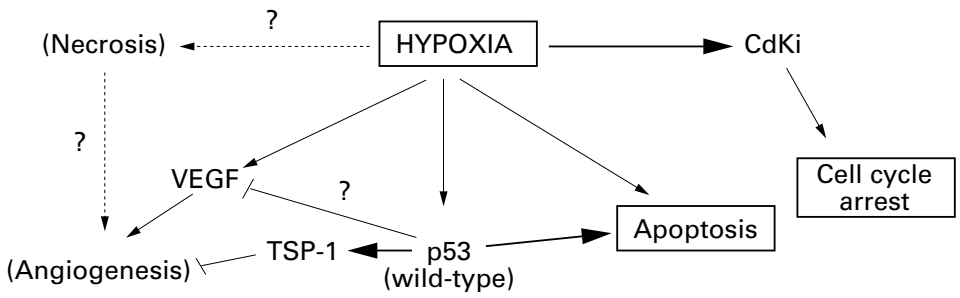

B

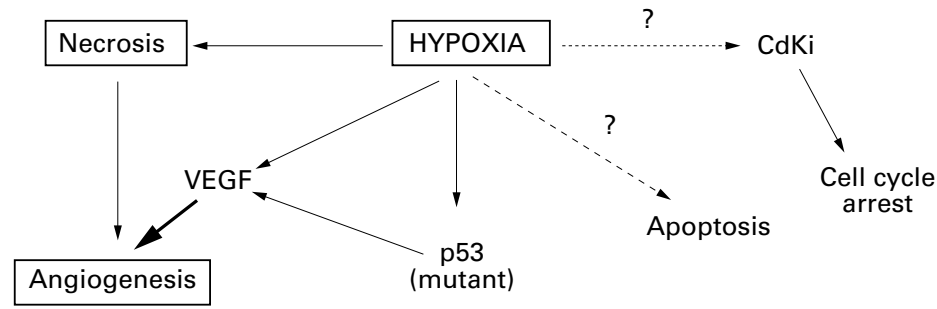

Figure 2 Comparison of hypoxia induced responses in normal $(A)$ and tumour $(B)$ cells. (A) Hypoxia induces cell cycle arrest and $p 53$ dependent apoptosis resulting in negligible necrosis. Angiogenesis is promoted by hypoxia because of induction of VEGF expression, but this is counterbalanced by production of wild-type p53, which in turn switches on expression of TSP-1. Homeostasis of the tissue is thus maintained and inappropriate angiogenesis does not occur. (B) In tumour cells bearing mutant p53, hypoxia induced VEGF is not so readily controlled by such anti-angiogenesis molecules as TSP-1, and neovascularisation occurs. Alternatively, apoptosis fails to occur and the resultant overproduction of tumour cells form areas of hypoxia and necrosis. More proangiogenic factors are then produced by the hypoxic tumour cells, which then induces a new blood supply in these areas. The cycle of cell activities is then repeated.

expression of such pro-VEGF (although the $\mathrm{B}$ and $\mathrm{C}$ forms of VEGF remain unaffected) and bFGF. ${ }^{19}{ }^{20}$ This upregulation is conferred by two distinct hypoxia driven mechanisms: one that is mediated by hif-1 binding to its specific 5 ' regulatory sequence on the VEGF promoter (the hre), and the other involving a 3 ' untranslated sequence of the VEGF gene that gives rise to VEGF mRNA stabilisation under hypoxia. ${ }^{21}$

Recent findings have shown that wild-type p53 upregulates the expression of the antiangiogenic agent, thrombospondin-1 (TSP-1). ${ }^{22}$ It may also act to downregulate VEGF expression, although a direct repressor function for p53 in hypoxic conditions has recently been shown to be unlikely (fig 2). ${ }^{23}{ }^{24}$ Wild-type p53 is also capable of blocking increases in the transcriptional activation of the VEGF gene induced by the activated form of Src, v-src. ${ }^{23}$ This report also showed that the p53 mutant, ala14 $\rightarrow$ val, appeared to upregulate VEGF expression. Recent findings suggest that the p53 mutant ala135 $\rightarrow$ val but not the wild-type protein stimulates the production of VEGF. This mutant p53 also potentiated the action of phorbol esters (TPA), which activate PKC and stimulate VEGF expression. Moreover this mutant p53 specifically increased phorbol ester production of VEGF and not other TPA inducible genes. ${ }^{25}$ However, we and others have shown that not all p53 mutations are equal, ${ }^{26}{ }^{27}$ and the effect of different p53 point mutations on VEGF production has yet to be ascertained. Plate et al, using immunohistochemistry, failed to demonstrate a correlation between p53 overexpression and VEGF induction in human glioma cells ${ }^{28}$; but several other reports have demonstrated a positive association between loss of p53 suppressor activity with induction of angiogenesis. ${ }^{29}$ Moreover, a correlation between VEGF expression and p53 stabilisation (presumably due at least in part to mutation) has been reported in lung cell tumours. ${ }^{26}$

Our preliminary studies on VEGF expression by breast tumour cell lines have shown that cells with p53 generally secrete very low levels of VEGF but that this rises approximately 25-fold under hypoxic conditions. Cell lines expressing mutant p53 release more VEGF in normoxia, and this rises even further under hypoxia (Royds JA, Lewis CE, unpublished data, 1997). This finding is supported by a recent report showing that the switch to an angiogenic phenotype in cultured fibroblasts from Li-Fraumeni patients coincided with hemizygosity for the mutant $\mathrm{p} 53$ allele. ${ }^{30}$

Our recent study of angiogenesis and p53 expression in more than 100 breast cancer biopsy specimens has shown that tumours with mutant p53 elicit a significantly stronger angiogenic response and the production of a denser final vasculature than tumours with a wild-type p53 gene. Patients with extensive p53 positivity in their tumours also had poorer survival. $^{31}$ Thus, the acquisition of a mutant p53 provided an enhanced angiogenic potential, and facilitated tumour progression and metastasis. This may not merely be the result of the loss of such p53 tumour suppressor functions as reduced TSP-1 expression, but also be caused by gain of function leading to the production of VEGF. Furthermore, it is likely that not all mutations of p53 are equal in their ability to promote tumour progression in this way.

\section{Molecular mechanisms involving NFkB: tumour cell responses to hypoxia}

Recently, evidence has emerged for a second hypoxia responsive pathway. The transcription factor, NFkB, has been shown to respond to a variety of metabolic stress signals including hypoxia. ${ }^{32}$ This heterodimeric protein is constitutively expressed in cells and, once activated, translocates from the cytoplasm to the nucleus to activate gene transcription. Most of the genes encoding cytokines that have been implicated in angiogenesis (for example, VEGF, basic fibroblast growth factor, bFGF, and tumour necrosis factor (TNF) $\alpha$ ) have NFkB binding sites in or near their promoters. Moreover, the expression of many of these cytokines are interlinked and co-regulatoryfor example, $\mathrm{TNF} \alpha$, induced by hypoxia in macrophages and other cells, stimulates production of such growth factors as bFGF by endothelial cells. ${ }^{33}$

NFkB can rapidly transduce hypoxic signals by increasing its DNA binding activity. This is highly regulated and facilitates the rapid control of many genes. An example of this is the effect of hypoxia on the binding of NFkB p65 (relA) to specific binding sites on the cyclooxygenase 2 (COX2) gene promoter in vascular endothelial cells. ${ }^{34}$ 
Many of the molecular mechanisms regulating, and regulated by, NFkB are known. It is a member of the Rel-related proteins (p50, p52, $\mathrm{v}$-Rel, c-Rel, RelA and B), which form dimeric DNA binding complexes at $\mathrm{kB}$ sites (GGGANNTTCC). Rel-related proteins form a large number of dimeric complexes, the most abundant of which is the strongly transactivating p50-p65 heterodimer. Inactive NFkB dimers exist in the cytoplasm through interactions with an inhibitor protein $\mathrm{IkB}$, a member of a family of regulatory proteins characterised by their multiple ankirin repeats and including $\mathrm{IkB}-\alpha, \mathrm{IkB}-\beta$, and $\mathrm{Bcl} 3$. The inactive trimer reacts to stimulatory signals by the targeted phosphorylation, and subsequent ubiquination and degradation of $\mathrm{IkBa}$, thus allowing the NFkB dimer to enter the nucleus and bind DNA. As protein synthesis is not required, genes that contain $\mathrm{NFkB}$ binding sites are able to undergo rapid expression following exposure to stimulatory signals such as cytokines. Genes regulated by $\mathrm{NFkB}$ include those involved in inflammatory responses and immune functions, such as those for the cell adhesion molecules VCAM, ICAM, and E selectin, and the cytokine/growth factors, interleukin (IL)-2, IL-6, IL-8, and G-CSF. Other genes under $\mathrm{NFkB}$ control include c-myc and the mutual regulation of $\mathrm{NFkB}$ and IkB.

Cytokine mediated NFkB activity is induced through serine phosphorylation of $\mathrm{IkBa}$ on residues 32 and 36, followed by proteolytic degradation of the inhibitor IkB, which unmasks the nuclear translocation signal on the NFkB dimers. DNA binding ensues with both homodimers and heterodimeric NFkB complexes containing p65 and p50 subunits, recognising a similar consensus sequence (GGGAATTTCC), enabling them to influence gene transcription. ${ }^{35}$

Alternatively, hypoxia and/or re-oxygenation induced NFkB activity has been shown to occur following tyrosine phosphorylation of the cytoplasmic inhibitory subunit IkBa on residue 42 , and does not involve proteolysis of the inhibitor IkB. ${ }^{36}$ Hypoxia does, however, lead to increased NFkB DNA binding activity and transactivation of $\kappa \mathrm{B}$ reporter constructs. ${ }^{28}$ The tyrosine phosphorylation of IkBa inhibits its phosphorylation on serine thus preventing the activation of $\mathrm{NFkB}$ via the ubiquinationdegradation route, thereby providing a proteolysis independent mechanism for $\mathrm{NFkB}$ activation. ${ }^{36}{ }^{37}$ Furthermore, it has been shown that activation of the proto-oncogene, src, occurs within 15 minutes of cellular exposure to hypoxia, and that the downstream effectors of src, namely ras and raf, are involved in the tyrosine activation pathway of NFkB. Thus it has been postulated that src activation is one of the earliest signalling events in the hypoxia response cascade leading to ras and raf-1 kinase activation, and ultimately to tyrosine phosphorylation of $\mathrm{IkB}$ and $\mathrm{NFkB}$ activation. ${ }^{32} 35$

The exact pathway regulating $\mathrm{NFkB}$ activity under hypoxic conditions has yet to be fully elucidated, but is possibly regulated by the type of NF $\kappa$ dimers activated. Only complexes that contain at least one of the strongly transactivating members of the Rel family (p65, etc) are efficiently regulated by $\mathrm{IkBa}$, and therefore cytoplasmic until activated. Homodimers of NFkB1-p50 or NFkB2-p52 may be controlled within the nucleus, as NFkB1-p50 and NFkB2-p52 subunits lack the IkB binding sequence and therefore cannot be retained in the cytoplasm. The p50 and p52 subunits also lack transactivation domains and probably serve as transcriptional repressors by masking DNA binding sites making them unavailable to the highly transactivating species of NFkB. The possibility that $\mathrm{p} 50$ and $\mathrm{p} 52$ can provide low levels of transcription cannot be ruled out. The p50, and possibly p52, homodimers can be targeted by the IkB related protein Bcl-3, which may be both agonists and antagonists of NFkB activity. Activated Bcl-3 can mediate positive transcriptional activation either by liberating $\mathrm{kB}$ sites occupied by inhibitory p50-p52 dimers or by converting inert complexes into transcriptionally active ones by virtue of its own transactivating domain. Bcl-3 was originally identified as a proto-oncogene because of its involvement in the 14:19 translocation characteristically found in chronic lymphocytic leukaemia; however, the $\mathrm{Bcl}-3$ protein has since been shown to be a member of the IkB family. Alternatively, it has been shown that transiently expressed Bcl-3 can have a negative transcriptional effect by inhibiting NFkB dependent expression in a dose dependent manner. Cell type and differentiation state may dictate which system is operational. This complex family of proteins generates remarkable flexibility for control of NFkB.

The association between IkB and NFkB is controlled by phosphorylation, which induces polyubiquination, targeting the molecule for destruction. Several kinases have been implicated in the upstream signalling from cytokine to $\mathrm{IkB}$, including $\mathrm{MEKK}-1$, a $700 \mathrm{~K}$ kinase complex that required ubiquination for activation, and $900 \mathrm{~K}$ complex (IKKa) similar to CHUK. These kinases result in specific SER32 and 36 phosphorylation. This latter enzyme, renamed IKKa, can also phosphorylate the specific serines in $\mathrm{IkBb}$ (ser 19 and 23). Moreover IKKa is deactivated by dephosphorylation by PP2A phosphatase, consistent with the observations that NFkB can be activated by inhibiting PP2A. Thus cytokine/stress activates a kinase (MEKK1 has not been shown to phosphorylate IKKa but only p700), which phosphorylates IKKa (p700), which phosphorylates IkB, which leads to the destruction of the inhibitor, and NFkB is activated. Dephosphorylation of IKKa by PP2A inhibits this pathway, and okadonic acid, which inhibits $\mathrm{PP} 2 \mathrm{~A}$, can activate $\mathrm{NFkB}$. The question arises as to whether $\mathrm{IKKa}$ is the unique integrator of the NFkB response or whether different stimuli involve different kinases. ${ }^{38}$

\section{Oncogenesis and NFkB}

Members of the NFkB family have been considered to be proto-oncogenes because subversion of their normal functions can lead to tumorigenesis. Thus NFkB2 (p52) and the 
Bcl3 genes are translocated in some lymphomas leading to oncogenic activation. The strongly transactivating $\mathrm{p} 65 / \mathrm{NFkB}$ is also activated by viral transforming proteins (Tax and LMP1), and antisense to p65/RelA blocks this transformation. IkB on the other hand is a possible tumour suppressor, and constitutive phosphorylation of IkB may have oncogenic activity by virtue of constant activation of NFkB regulated genes. This constitutive activation of $\mathrm{NFkB}$ is also seen in IkB deficient mice. Moreover, antisense IkBa treatment of cells results in oncogenic transformation. ${ }^{39} \mathrm{It}$ is of interest that NFkB is activated in macrophages by the antineoplastic agent paclitaxel (Taxol; Bristol Myers Squibb, Middlesex, UK) contributing to this agent's antitumour properties. $^{40}$

\section{Role of NFkB in hypoxia induced p53 expression}

Wu and Lozano, in 1994, were the first to report that $\mathrm{NFkB}$ regulates p53 gene expression. $^{41}$ The role of $\mathrm{NFkB}$ in $\mathrm{p} 53$ promoter activation is not fully understood but the region of the p53 promoter required to respond to genotoxic stress extends from -70 to -40 , which overlaps the NFkB site. ${ }^{42}$ The NFkB motif in the p53 promoter has a high affinity for NFkB, and has been shown to function as a promoter element when placed artificially upstream of TATA elements. Thus, NFkB family members may play a role in p53 regulation in response to certain types of stress. Indirect evidence for this has recently been provided in molecular studies of the ataxia telangiectasia (AT) gene. NFkB-IkB has been shown to contribute to the AT phenotype, acting downstream of the primary genetic defect. p53 is also thought to act downstream of the AT gene product and may, therefore, be at least one of the targets for NFkB control of this gene. $^{43}$

Genotoxic stress induces p53 expression resulting in $\mathrm{G}_{1}$ arrest but the role of $\mathrm{NFkB}$ as a transcription factor in p53 regulation under these conditions is dubious. ${ }^{44}$ However, Baldwin $^{45}$ showed that p53 mRNA was induced by a non-genotoxic stress-hypoxia. Hypoxia probably induces p53 activity via a different pathway from DNA damaging agents as hypoxia but not ionising radiation caused p53 accumulation in the presence of the E6 viral protein. Moreover, $G_{1}$ arrest following hypoxia is similar in wild-type and mutant p53 cells. ${ }^{16}$ The relatively quick reversal of $\mathrm{p} 53$ protein concentrations following re-oxygenation also argues in favour of an active role for p53 in hypoxic stress. However, as the hypoxia induced $G_{1}$ arrest appears to be independent of the p53 genotype, it is likely that p53 mediates some function other than $G_{1}$ arrest in response to hypoxia, possibly involving control of apoptosis and/or angiogenesis. Long term hypoxia is expected to result in genotoxic damage, and p53 may have a role in tumour suppression under such conditions, possibly involving GADD45 induction. DNA binding activity of hypoxia induced p53 was competed out by a self oligo and a GADD45-p53 oligo, but not by an unrelated NFkB oligo. ${ }^{16}$

The NFkB element in the p53 promoter has a high affinity for NFkB p50 homodimers, but it is not transactivated by $\mathrm{p} 65$ or inhibited by IkB. ${ }^{42}$ This suggests a nuclear rather than a cytoplasmic regulation of $\mathrm{p} 53$ by $\mathrm{NFkB}$. This is contradictory to the findings of $\mathrm{Wu}$ and Lozano who showed p53 activation following the tyrosine phosphorylation of $\mathrm{IkBa} .^{41}$ The reason for this discrepancy is not clear; however, the fact that these different studies used different species (murine $v$ human) and employed different culture conditions (serum $v$ serum free) means that the results are not strictly comparable.

A nuclear rather than cytoplasmic regulation of NFkB activation in response to hypoxia is also suggested by the finding that Ras and Raf activation leads to gene transcription at NFkB sites without nuclear translocation of $\mathrm{NFkB}$. The mechanism is unclear but may involve the stimulation of the constitutively nuclear forms of NfkB. ${ }^{44}$ Ras and Raf work downstream from membrane tyrosine kinases such as src, which is activated by low oxygen conditions. ${ }^{46}$ Most importantly wild-type p53 action can override the angiogenic effects of the activated oncogene v-src. Wild-type p53 prevented v-src from activating transcription of the VEGF promoter. This suggests that $\mathrm{p} 53$ activation by hypoxia has an antiangiogenic role, possibly by induction of TSP-1 and inhibition of VEGF. ${ }^{23} \mathrm{~A}$ direct role for Ras in modulating VEGF activity has been proposed, thus linking the angiogenic response to hypoxia with Ras activation, although VEGF induction itself is thought to be via the Hif-1 pathway. ${ }^{47}$ The antitumour activity of the chymotrypsin-like protease inhibitor, TPCK, acts by blocking Ha-ras induced transcriptional activity of NFkB while having little affect on the ability of Ha-ras to activate AP-1 transcription. ${ }^{48}$ Thus the two hypoxia response pathways mediated by Hif-1 and NFkB may interact to provide tight control of angiogenesis in vivo.

Regulation of $\mathrm{p} 53$ expression in response to hypoxia may embrace both cytoplasmic control via tyrosine phosphorylation of $\mathrm{IkB}$ and nuclear control. Thus the nuclear and cytoplasmic regulation of Rel-domain transcription factors may complement each other and provide a remarkably flexible system of control for transcriptional activation of many genes involved in the hypoxic response including TNF $\alpha{ }^{16}$ TNF $\alpha$ is a cytokine produced by many cell types including macrophages and some tumour cells in response to environmental stress such as hypoxia; it elicits a spectrum of responses including apoptosis. TNF binding to its receptor induces apoptosis and activates $\mathrm{NFkB}$ via a signal transduction cascade, which may involve raf- 1 and activation of IkB kinase. $\mathrm{TNF} \alpha$ induced breakdown of $\mathrm{IkBa}$ involves ubiquination of the amino terminal signal response domain (SRD) followed by degradation of the protein. Overexpression of NFkB or its activation by IL-1 protects against TNF induced apoptosis, possibly by induction of 
anti-apoptotic genes. ${ }^{49}$ Inhibition of $\mathrm{NFkB} / \mathrm{Rel}$ activity can induce spontaneous apoptosis in $\mathrm{B}$ cells. ${ }^{50}$

$\mathrm{TNF} \alpha$ has also been shown to promote NFkB binding to the $\mathrm{p} 53$ promoter in vitro ${ }^{49}$ and we have shown a correlation between macrophage $\mathrm{TNF} \alpha$ concentrations and $\mathrm{p} 53$ expression in breast cancer biopsy specimens. ${ }^{31} \mathrm{At}$ least seven categories of genes have been shown to be transcriptionally regulated by NFkB: cell adhesion molecules, certain proto-oncogenes (such as c-myc), cytokines (and their receptors), matrix proteins, matrix degrading enzymes, as well as genes modulating apoptotic sensitivity, which may include p53.

\section{Conclusions}

NFkB transactivation is a downstream effector of hypoxia/reoxygenation, possibly involving, either singly or in combination, tyrosine phosphorylation of $\mathrm{IkBa}$ and nuclear control of $\mathrm{NFkB}$ by $\mathrm{p} 50$ and Bcl3. Phosphatases may also play a role in activation of NFkB. The mechanism of p53 induction as a result of hypoxia/reoxygenation differs from that for genotoxic insult and probably involves the NFkB family of transcription factors. A cell specific mode of signalling and regulation in response to hypoxia/reoxygenation should be considered, as should the possibility of functioning autoregulatory loops. Different responses may occur in response to different doses of gene product. Factors such as these may account for some of the apparently conflicting findings, which are inevitable in this relatively new branch of molecular tumour pathology.

Activation of $\mathrm{p} 53$ transcription in response to hypoxia/reoxygenation by NFkB may be by the nuclear rather than the cytoplasmic control mechanism, mediated by upstream factors such as src, ras, raf, $\mathrm{PKCz}$, and stress activated protein kineses. The $\mathrm{p} 53$ accumulation kinetics are slower for hypoxia than ionising radiation, and its homeostatic role in hypoxia is probably not to induce $G_{1}$ arrest as this function is overridden by other cell cycle control factors. A more likely role for p53 in hypoxia is in the induction of apoptosis when oxygen tension falls below $0.2 \%$, and in the control of angiogenesis. The possibility that NFkB may also influence p53 gene expression indirectlyfor example, via c-myc expression, should be considered.

Obviously much more work is required to elucidate the role of $\mathrm{NFkB}$ in the hypoxia signalling pathway, which in turn will enable us to devise a therapeutic approach to cancer based on a new concept of tumour hypoxia as a strategic tool by which tumour cells can be made more susceptible to drugs and/or hypoxia than normal cells. By understanding the signal transduction pathways mediating the effect of hypoxia in tumour cells, it may be possible to manipulate these for therapeutic gain.

We thank Ms K Webley and Mr K Corke for their contributions to this work. The authors also gratefully acknowledge the to this work. The authors also gratefully acknowledge the support of the: YCRC, EPSRC, Special Trustees of the Former Breast Cancer Campaign, and Special Trustees of the Former
United Sheffield Hospitals (CEL); the Wellcome Trust, MRC Nuffield Foundation, BBSRC, The Meningitis Trust (SKD); Special Trustees of the Former United Sheffield Hospitals, British Heart Foundation, ARC (EEQ).

1 Vaupel PW. The influence of tumour blood flow and microenvironmental factors on the efficacy of radiation, drugs and localised hyperthermia. Klin Padiatr 1997;209:243-49.

2 Gimbione MA Jr, Leapman SB, Cotran RS, et al. Tumour domancy in vitro by prevention of neovascularization. $f$ domancy in vitro by preve
Exp Med 1972;136:261-76.

3 Jain R. Vascular and interstitial physiology of tumours: role in cancer detection and treatment. In: Lewis CE, Bicknell , Ferrara N, eds. Tumour angiogenesis. Oxford: Oxford University Press, 1997;45-60.

4 Leek RD, Harris AL, Lewis CE. Cytokine netwoorks in solid human tumours: regulation of angiogenesis. $\mathcal{F}$ Leuk Biol 1994;56:423-35.

5 Brown LF. Expression of vascular permeability factor (VEGF) and its receptors in breast cancer. Hum Pathol 1995;26:86-91.

6 Zhang H-T, Craft P, Scott PAE, et al. Enhancement of tumour growth and vascular density by transfection of vascarcinoma cells. F Natl Cancer Inst 1995;87:213-19.

7 Melnyk O, Shuman MA, Kim KJ. Vascular endothelial growth factor promotes tumour dissemination by a mechgrowth factor promotes tumour dissemination by a mech-
anism distinct from its effect on primary tumour growth. anism distinct from its eff

8 Carmeliet P, Ferreir V, Breier G, et al. Abnormal blood vessel development and lethality in embryos lacking a single VEGF allele. Nature 1996;380:435-9.

9 Vincenti V, Cassano C, Rocchi M, et al. Assignment of the vascular endothelial growth-factor gene to humanchromosome 6P21.3. Circulation 1996;93:1493-5.

10 Shimizu S, Eguchi Y, Kamiike W, et al. Induction of apoptosis as well as necrosis by hypoxia and predominant prevention of apoptosis by Bcl-2 and $\mathrm{Bcl}-\mathrm{X}_{\mathrm{L}}$. Cancer Res 1996;56: 2161-6.

11 Long X, Boluyt MO, Hipolito MdeL, et al. P53 and the hypoxia-induced apoptosis of cultured neonatal rat cardiac mypoxia-induced apoptosis of cultured neonates.

12 Wang GL, Jiang BH, Rue EA, et al. Hypoxia-inducible factor 1 is a basic helix-loop-helix-PAS heterodimer regulated by cellular O2 tension. Proc Natl Acad Sci USA 1995;92: 5510-14

13 Semenza GL, Wang GL. A nuclear factor induced by hypoxia via de novo protein synthesis binds to the human erythropoetin gene in transgenic mice. Mol Cell Biol 1992; 12:5447-54

14 Masatsugu E, Shinichiro T, Noboru Y, et al. A novel bHLHPAS factor with close sequence similarity to hypoxiainducible factor la regulates the VEGF expression and its potentially involved in lung and vascular development. Proc Natl Acad Sci USA 1997;94:4273-8.

15 Tian H, McKnight SL, Russell DW. Endothelial PAS domain protein 1 (EPAS1), atranscription factor selectively expressed in endothelial cells. Genes Dev 1997;11:72-82.

16 Graeber TG, Peterson JF, Tsai M. Hypoxia induces the accumulation of p53 protein but the activation of a G1-phase checkpoint by low oxygen conditions is independent of p53 status. Mol Cell Biol 1994;14:6264-77.

7 Kim CY, Tsai MH, Osmanian C, et al. Selection of human cervical epithelial cells that possess reduced apoptotic potential to low oxygen conditions. Cancer Res 1997;57: $4200-4$

18 Titsy T. A p53 mt provides a gain-of-function phenotype affecting cell cycle checkpoint control. Proceedings of the 8th international p53 workshop. Dundee, 1996.

19 Enholm B, Paavonen K, Ristimaki A, et al. Comparison of EEGF, VEGF-B, VEGF-C and Ang-1 mRNA regulation by serum, growth factors, oncoproteins and hypoxia. Oncogene 1997;14:2475-83.

20 Kuwabara K, Ogawa S, Matsumoto M, et al. Hypoxiamdiated induction of acidic/basic FGF and PDGF in mononuclear phagocytes stimulates growth of hypoxic endothelial cells. Proc Natl Acad Sci USA 1995;92:460610.

21 Damert A, Machein M, Breier G, et al. Upregulation of VEGF expression in a rat glioma is conferred by two distinct hypoxia-driven mechanisms. Cancer Res 1997;57: 3860-4.

22 Dameron KM, Volpert OV, Twainsky MA, et al. Control of angiogenesis in fibroblasts by $\mathrm{p} 53$ regulation of thrombospondin 1. Science 1994;265:1582-4.

23 Mukhopadhyay D, Tsiokas L, Sukhatme VP. Wild-type p53 and v-src exert opposing influences on human VEGF gene and v-src exert opposing influences on hun

24 Agani F, Kirsch DG, Friedman SL, et al. P53 does not repress hypoxia-induced transcription of the VEGF gene. Cancer Res 1997;57:4474-7.

25 Keiser A, Weich HA, Brandner G, et al. Mt p53 potentiates PKC induction of vascular endothelial growth factor expression. Oncogene 1994;9:963-9.

26 Rolley N, Butcher S, Milner J. Specific DNA binding by different classes of human p53 mutants. Oncogene 1995;11: 763-70.

27 Webley KM, Royds JA. Effect of mutation and conformation on the function of the tumour suppressor gene p53 in tion on the function of the tumour supp
colorectal cancer. F Pathol. [In press.]

28 Plate KH, Breir G, Weich HA, et al. VEGF and glioma angiogenesis. Int 7 Cancer 1994;59:520-9. 
29 Van Meier EG, Polverini PJ, Chazin VR, et al. Release of an inhibitor of angiogenesis upon induction of wild type p53

30 Fontanini G, Vignati S, Mussi A, et al. Neoangiogenesis and p53 protein in lung cancer: their prognostic role and their relation with vascular endothelial growth factor (VEGF) expression. Br f Cancer 1997;75:1295-301.

31 Royds JA, Lewis CE, Leek RD, et al. Association of $\mathrm{p} 53$ status with tumour angiogenesis, grade, necrosis and macrophage activity in invasive breast cancer [abstract]. F Pathol 1997;182:17A

32 Koong AC, Chen KY, Giaccia AJ. Hypoxia causes the activation of nuclear factor kappa through the phosphorylation of IKBa on tyrosine residues. Cancer Res 1994; 54:1425-30.

33 Samaniego F, Markham PD, Gendelman R, et al. Inflammatory cytokines induce endothelial cells to produce and release bFGF and to promote Kaposi's sarcoma-like lesions in nude mice. F Immunol 1997;158:1887-94.

34 Schmedtje JF, Ji YS, Liu W, et al. Hypoxia induces cyclooxygenase-2 via the NFkB p65 transcription factor in cyclooxygenase-2 via the NFkB p65 transcription factor in human

35 DiDonato J, Mercurio F, Rosette C, et al. Mapping of the sites that signal its ubiquination and degradation. Mol Cell Biol 1996;16:129554.

36 Imbert V, Rupec RA, Livolsi A, et al. Tyrosine phosphorylation of IkB activates $\mathrm{NFkB}$ without proteolytic degradation of IkB. Cell 1996;86:787-98.

37 Singh S, Darnay BG, Aggarwal BB, et al. Site-specific tyrosine phosphorylation of $\mathrm{IkB}$ negatively regulates its inducible phosphorylation and degradation. $7 \mathrm{Biol}$ Chem 1996;271:31049-54.

38 Isreal A. IkB all zipped up. Nature 1997;388:519-21.

39 Beauparlant P, Kwan I, Bitar R, et al. Disruption of I-Kappa-B-alpha regulation by antisense RNA expression leads to malignant transformation. Oncogene 1994;9:318997.
40 Hwang S, Ding A. Macrophages derived from C3H/HEJ (LPS(D)) mice respond to bacterial lipopolysaccharide by activating NF-Kappa-B Cancer Biochem Biophys 1995;14: 265-72.

41 Wu H, Lozano G. NFkB activation of p53. I Biol Chem 1994;269:20067-74.

42 Sun X, Shimizu H, Yamamoto K-I. Identification of a novel p53 promoter element involved in genotoxic stressinducible p53 gene expression. Mol Cell Biol 1995;15: inducible

43 Jung $\mathrm{M}$, Zhang Y, Lee S, et al. Correction of radiation senstivity in ataxia telangiectasia cells by a truncated IkB- $\alpha$. Science 1995;268:1619-21.

44 Tuck SP, Crawford L. Characterisation of the human p53 gene promoter. Mol Cell Biol 1989;9:2163-72.

45 Baldwin AS. The NFkB and IkB proteins: new discoveries and insights. Ann Rev Immunol 1996;14:649-83.

46 Koong AC, Chen KY, Mivechi NF, et al. Hypoxic activation of nuclear factor $\mathrm{K} \mathrm{B}$ is mediated by a ras and raf signalling pathway and does not involve MAP kinase (ERK1 or ERK2). Cancer Res 1994;54:5273-9.

47 Mazure NM, Chen KY, Yeh P, et al. Oncogenic transformation and hypoxia synergistically act to modulate VEGF expression. Cancer Res 1996;56:3436-40.

48 Denko NC, Chen KY, Laderoute KR, et al. Protease inhibitor TPCK represses Ha-ras (Val-12) transformation and nuclear factor-kappa B activation. Int f Oncol 1997;10: 895-900.

49 Liu Z, Hsu H, Goeddel DV, et al. Dissection of TNF receptor 1 effector functions: JNK activation is not linked to apoptosis while NFkB activation prevents cell death. Cell 1996;87:565-76.

50 Wu GH, Havyuong L, Bellas RE, et al. Inhibition of NFkB/ Rel induces apoptosis in murine B cells. EMBO f 1996;15: 4682-90. 